\title{
Sports and Exercise Medicine
}

Open Access

CASE REPORT

\section{First Rib Fracture Nonunion Treated With Bone Marrow Aspirate Concentrate in a Division 1 Collegiate Baseball Player}

\author{
Kanjiya $S^{1 *}$, Gambardella $R A^{1}$ and Moon $C N^{2}$ \\ ${ }^{1}$ Cedars-Sinai Kerlan-Jobe Institute, USA \\ ${ }^{2}$ Director of Orthopaedic Trauma, Department of Orthopedics, USA
}

*Corresponding author: Shrey M. Kanjiya, MD, Cedars-Sinai Kerlan-Jobe Institute, USA

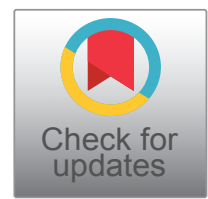

\section{Introduction}

Fractures of the first rib have historically been described in major trauma with a high prevalence for cardiothoracic and musculoskeletal fractures. However, there have been several case reports describing first rib fractures in athletes. The vast majority of these patients return to play with conservative management. Unfortunately, some patients may develop fracture nonunion. Patients that develop a nonunion may remain asymptomatic. In the rare case that a patient is symptomatic or develops thoracic outlet from a fracture nonunion then a first rib resection may be indicated. With the use of biologic agents such as bone marrow aspirate concentrate, physicians may have the option to treat first rib fracture nonunion without invasive procedures.

\section{Case Report}

A 19-year-old right hand dominant male division 1 collegiate baseball player presented to the office with 4 months of posterior shoulder pain. The pain began after colliding with the outfield wall will attempting to catch a ball. After this event he continued to play with mild posterior shoulder discomfort that was treated with physical therapy and symptomatic control. 2 weeks prior to presentation the patient complained of increased sharp pain after hitting a ball while batting in a game. On physical examination, he was found to have full range of motion and normal strength of the right upper extremity. He was minimally tender to palpation along the medial border of the scapula, trapezius, and rhom- boids. He had normal neurologic and vascular evaluation. The remainder of his shoulder exam has normal. Conventional shoulder radiographs were obtained in the office which demonstrated an acute non displaced posterior first rib fracture (Figure 1). The patient was treated conservatively and discontinued playing baseball. Evaluation 6 weeks later demonstrated normal physical exam findings and radiographs showed early callus formation. At 12 weeks ( 7 months from injury) from presentation the patient was allowed to resume baseball related activities. However, the patient began to have dull posterior shoulder pain with batting. A repeat conventional radiograph at that time indicated a possible fracture nonunion (Figure 2). A Computerized Tomography (CT) scan of the first rib was obtained, which confirmed a nonunion without evidence of bridging callus (Figure 3 ). After discussing additional treatment options, the patient elected to proceed with CT guided injection of Bone Marrow Aspirate Concentrate (BMAC). Under general anesthesia, 50cc of bone marrow was aspirated from the posterior right iliac crest (Figure 4). The bone marrow was centrifuged to yield approximately $7.5 \mathrm{cc}$ of BMAC. The BMAC was injected under $\mathrm{CT}$ guidance along the anterior aspect of the first rib fracture site (Figure 5). The patient was instructed to refrain from any therapy or baseball related activities. At 8 weeks post BMAC injection, a CT scan of the first rib was obtained which showed complete osseous union with bridging callus (Figure 6). At 12 weeks post BMAC injection, the patient was allowed to return to

Citation: Kanjiya S, Gambardella RA, Moon CN (2021) First Rib Fracture Nonunion Treated With Bone Marrow Aspirate Concentrate in a Division 1 Collegiate Baseball Player. Int J Sports Exerc Med 7:187. doi.org/10.23937/2469-5718/1510187

Accepted: April 15, 2021; Published: April 17, 2021

Copyright: (c) 2021 Kanjiya S, et al. This is an open-access article distributed under the terms of the Creative Commons Attribution License, which permits unrestricted use, distribution, and reproduction in any medium, provided the original author and source are credited. 


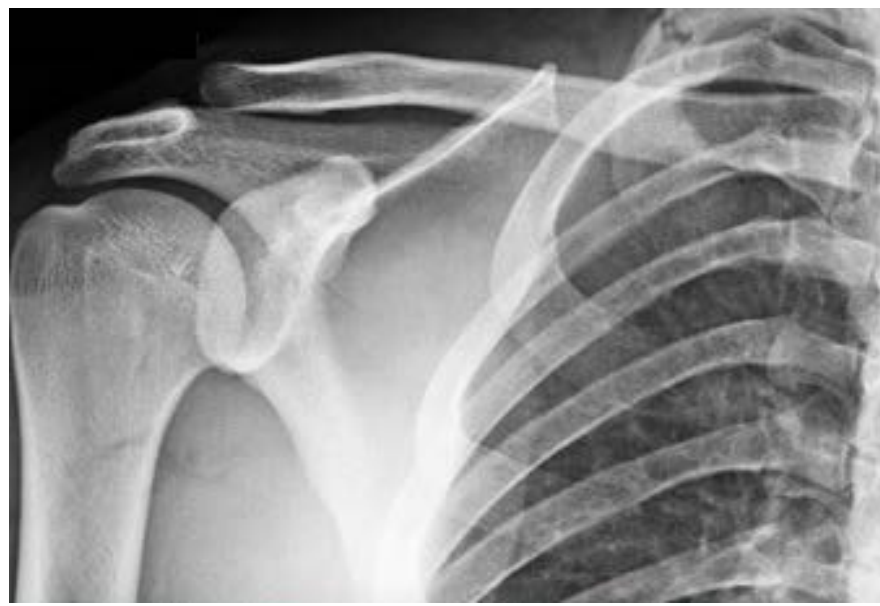

Figure 1: Anterior-posterior radiograph of the right shoulder which demonstrates a nondisplaced first rib fracture.

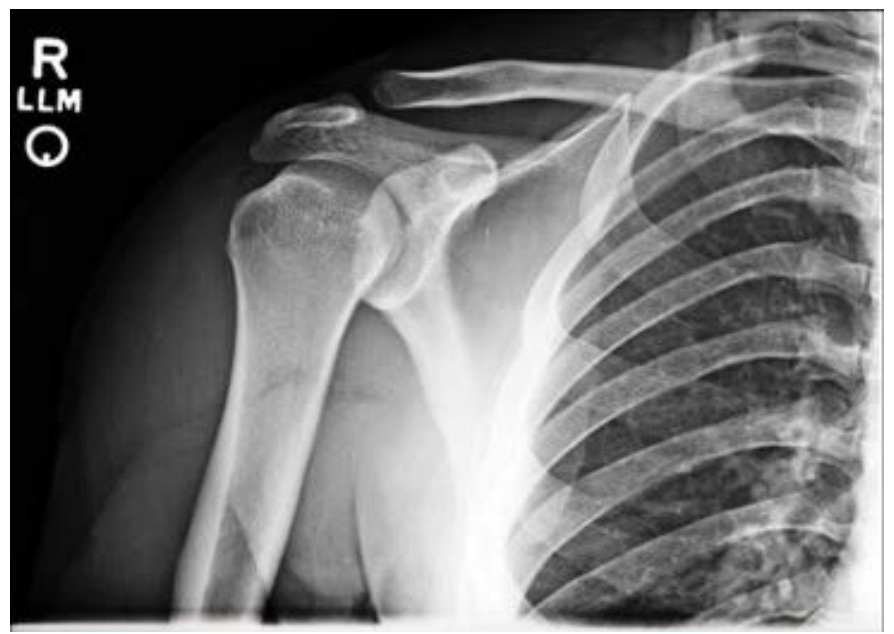

Figure 2: Anterior-posterior radiograph of the right shoulder which demonstrates a first rib fracture without evidence of bridging callus.

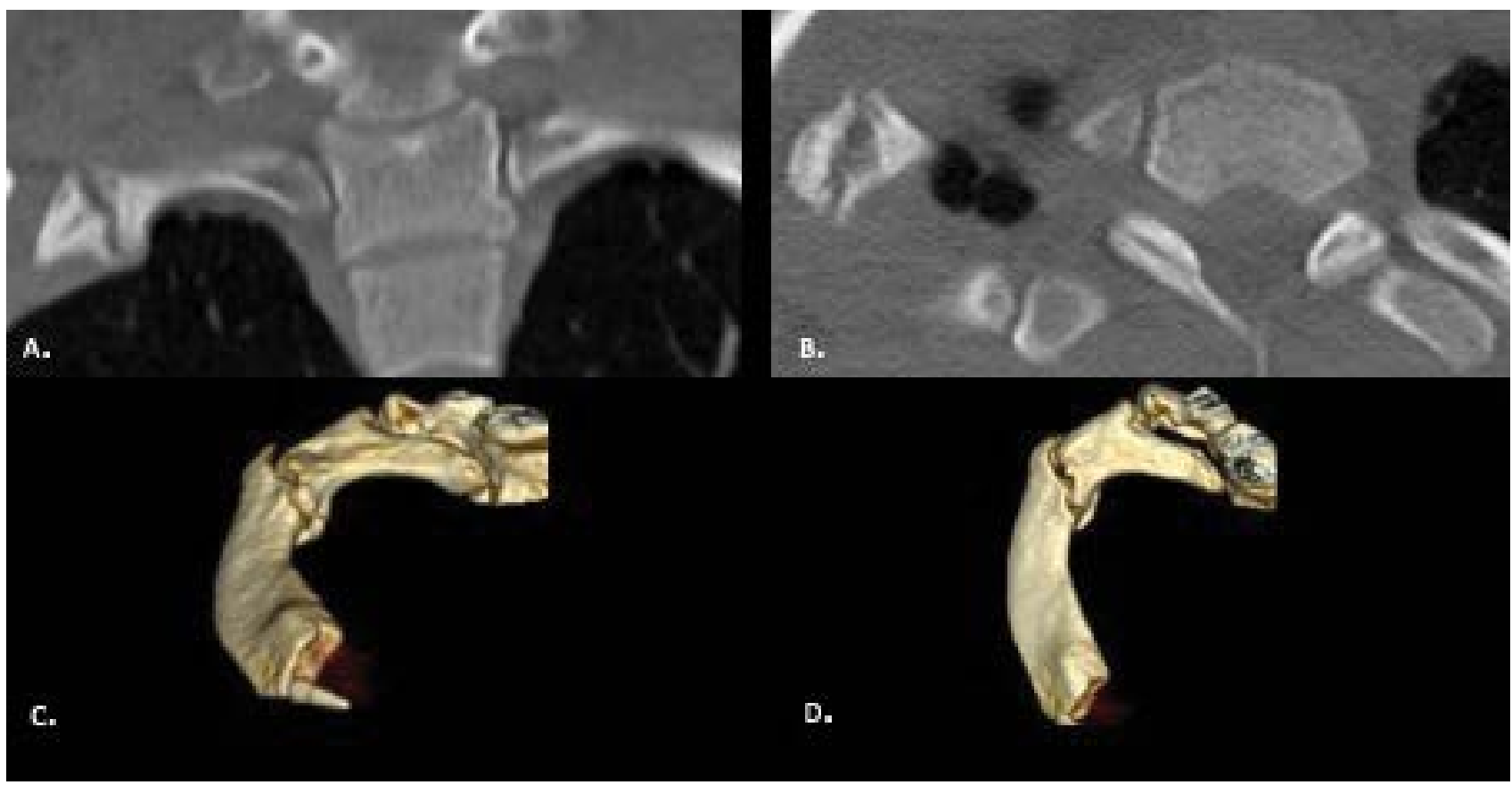

Figure 3: (A) Coronal; $(B)$ axial; (C and D) 3D reconstruction of the right first rib which demonstrates a nonunion of the first-rib. 


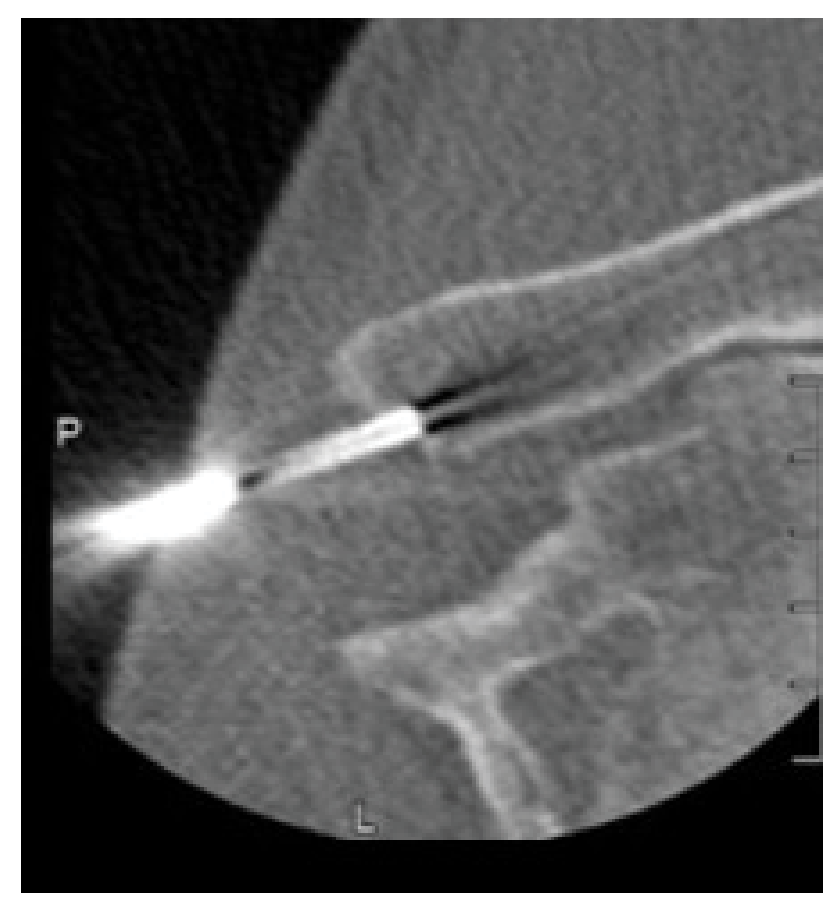

Figure 4: CT guided aspiration of bone marrow from the right posterior iliac crest.

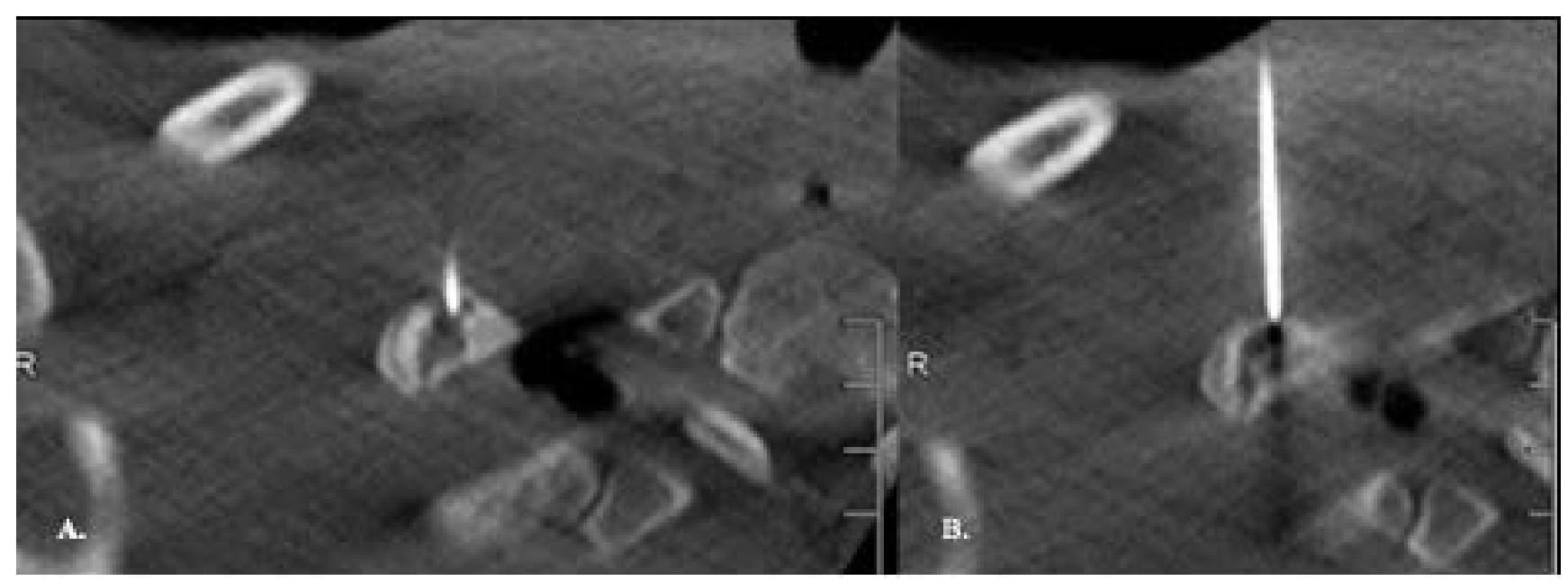

Figures 5A and 5B: CT guided injection of bone marrow aspirate concentrate into the anterior aspect of the right firstrib fracture site.

baseball related activities. The patient remained asymptomatic and successfully completed the remainder of the baseball season.

\section{Discussion}

Fractures of the first rib have historically been described in major trauma with a high prevalence for cardiothoracic and musculoskeletal fractures [1]. Isolated first rib fractures are exceedingly rare but have been described in athletes involved in overhead activities [25]. Funakoshi et al. retrospectively evaluated 24 first rib fractures in 23 overhead throwing athletes. They found that the majority $(19 / 24)$ of injuries occurred in the dominant arm and presented with acute shoulder pain after batting or pitching. After an average of 7.5 months with conservative management, 17 fractures (71\%) healed and 7 fractures (29\%) had a fracture nonunion.
3 athletes developed thoracic outlet syndrome and required first rib resection. In addition, they classified first rib fractures depending on the location of the fracture. Fractures can be located along the sub clavian groove, inside the middle scalene insertion, or behind the costo vertebral articulation [2].

Stress fractures of the first rib typically occur at the deep groove of the subclavian artery between the anterior and middle scalene muscles [6]. This section of the first rib is the thinnest and endures high tensile force from superior pull of the scalene muscles and inferior pull of the serratus anterior and intercostal muscles [6]. Repetitive motions that activate these muscles can result in stress fractures that may go on to complete fractures $[3,6,7]$.

First rib fractures generally have a good prognosis and can be treated with rest, activity modification, and gradu- 


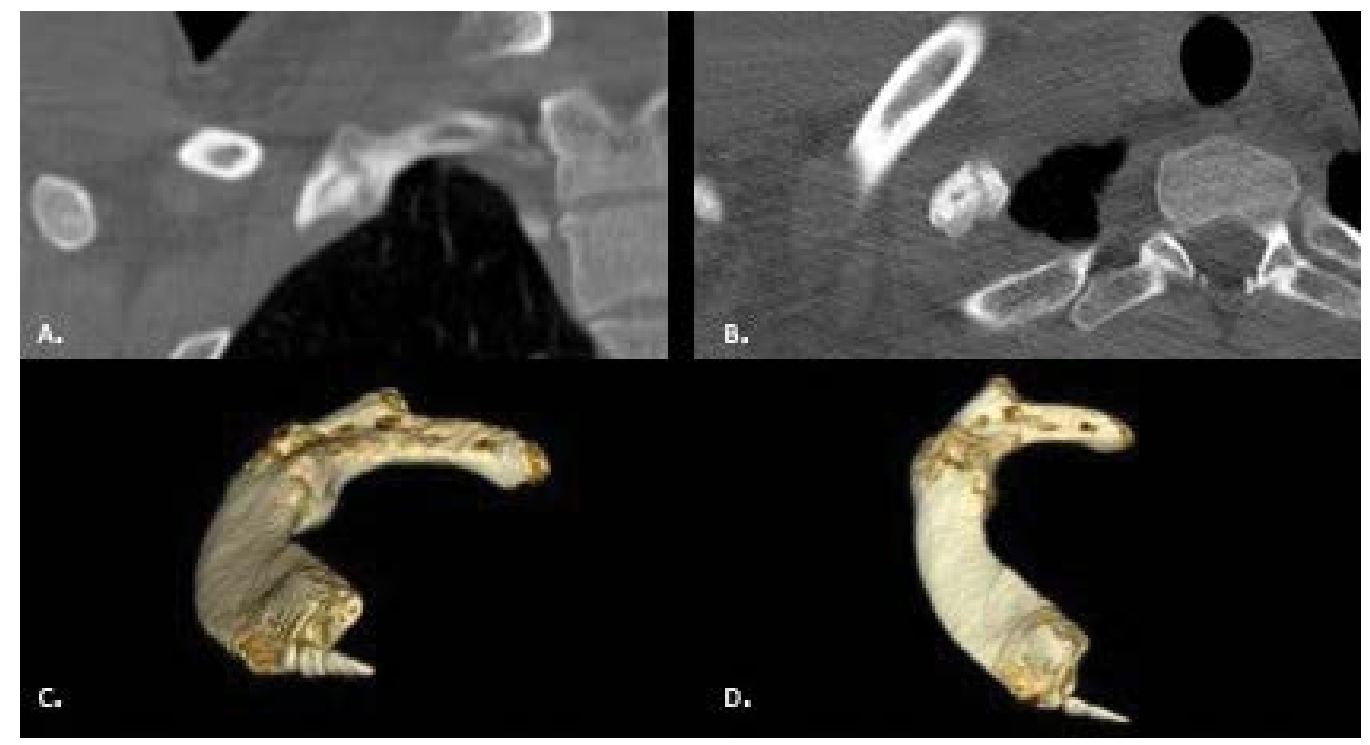

Figure 6: $(A)$ Coronal; $(B)$ axial; $(C$ and $D) 3 D$ reconstruction of the right first rib which demonstrates bridging callus and osseous union.

al return to play $[2,3,5]$. However, our patient developed a symptomatic nonunion of the first rib. There are few case reports of first rib nonunion [8-10]. In two of these case reports the patients developed thoracic outlet syndrome which required a first rib resection. In addition, Funakoshi, et al. had 3 patients (3/24) develop thoracic outlet syndrome that required a first rib resection [2].

Although our patient did not have signs or symptoms of thoracic outlet syndrome, he had continued posterior shoulder pain and was unable to participate in any baseball related activities despite adequate rest and rehabilitation. Our patient's fracture location was consistent with a stress fracture anatomically, but he did have a traumatic event. To avoid a first rib resection, we proposed the use of bone marrow aspirate concentrate via CT guided injection into the fracture site.

The patients fracture morphology was consistent with a hypertrophic nonunion. Classical treatment for a hypertrophic nonunion is to add stability. Typically, biology is not the issue, but rather biomechanical instability. However, there are no reports of repairing first rib fracture nonunion surgically. BMAC contains osteogenic progenitor cells and has been suggested as an autologous biologic adjunct to promote bone healing [11,12]. There are several clinical and animal studies that demonstrate the ability of BMAC to promote fracture union $[2,4,5,7,8]$. Gianakos, et al. performed a systematic review looking at BMAC in animal long bone healing [5]. They found 35 studies that met their inclusion criteria. Of studies reporting statistics, $100 \%$ showed increase in bone formation in the BMAC group on plain radiographs compared to control groups.

In addition to animal studies, there are several human clinical studies that demonstrate the osteogenic potential of BMAC in fracture nonunion or delayed union [11-15]. Garg, et al. reported bone marrow aspirate in- jection into 20 patients with ununited long bone fractures. $85 \%$ of patients $(17 / 20)$ went on to fracture union without further intervention [14]. Similarly, Hernigou, et al. reported 60 patients with tibial nonunion that were treated with BMAC [12]. They found that $88 \%$ of their patients obtained union without any further intervention. However, there are no clinical reports or studies that have used BMAC for first rib nonunion.

Our patient likely sustained a first rib stress fracture that likely completed during an acute event while playing baseball. While the majority of these injuries have a good prognosis and can be managed non operatively, our patient developed a fracture nonunion and continued to have pain. The options for nonunion management are scarce. We proposed CT guided injection of BMAC, a less invasive procedure that would prevent the need for surgery and allow the patient to return to play. He was successfully treated with CT guided BMAC injection into the first rib fracture site. Fracture union was achieved by 3 months and the patient returned to playing Division 1 collegiate baseball. He remained asymptomatic through the remainder of the baseball season.

\section{Conclusion}

Isolated first rib fractures can occur in overhead athletes. The majority of these patients can be treated with rest, activity modification, and gradual return to sports. However, a small subset of patients will develop fracture nonunion. Of those that remain symptomatic from fracture nonunion, the options for management are limited. First-rib resection has been described in the literature for treatment of symptomatic fracture nonunion or thoracic outlet syndrome secondary to fracture nonunion. Alternatively, we present a case where CT guided injection of BMAC can be used as an alternative form of treatment in patients with symptomatic nonunion without signs or symptoms of thoracic outlet syndrome. 


\section{References}

1. Richardson JD, McElvein RB, Trinkle JK (1975) First Rib Fracture: A Hallmark of Severe Trauma. Ann Surg 181: 251-254.

2. Funakoshi $\mathrm{T}$, Furushima $\mathrm{K}$, Kusano $\mathrm{H}$, Itoh $\mathrm{Y}$, Miyamoto $\mathrm{A}$, et al. (2019) First-Rib Stress Fracture in Overhead Throwing Athletes. J Bone Joint Surg Am 101: 896-903.

3. Kawashima K, Terabayashi N, Asano H, Akiyama H (2020) Classification of stress fractures of the first rib in adolescent athletes. J Pediatr Orthop B 29: 409-411.

4. Low S, Kern M, Atanda A (2016) First-rib stress fracture in two adolescent swimmers: a case report. J Sports Sci 34: 1266-1270.

5. Young BL, Watson SL, Meyer RD, Ponce BA (2016) Nonunion of first rib fracture in a softball player: case report of a rare cause of thoracic outlet syndrome. J Shoulder Elbow Surg 25: 353-357.

6. Mintz AC, Albano A, Reisdorff EJ, Choe KA, Lillegard W (1990) Stress fracture of the first rib from serratus anterior tension: an unusual mechanism of injury. Ann Emerg Med 19: 411-414.

7. Kawashima K, Terabayashi N, Miyagawa T, Tanaka R, Ogawa H, et al. (2016) Stress Fractures of the First Rib Related to Swinging of a Baseball Bat: Two Case Reports. Clin J Sport Med 26: 108-110.
8. Proffer DS, Patton JJ, Jackson DW (1991) Nonunion of a first rib fracture in a gymnast. Am J Sports Med 19: 198-201.

9. Puttmann KT, Satiani B, Vaccaro P (2016) Thoracic Outlet Syndrome in a Volleyball Player Due to Nonunion of the First Rib Fracture. Vasc Endovascular Surg 50: 563-565.

10. Young EJ, Curtis RJ (2008) First-rib stress fractures related to hitting in two baseball teammates. Clin J Sport Med 18: 300-301.

11. Gianakos A, Ni A, Zambrana L, Kennedy JG, Lane JM (2016) Bone Marrow Aspirate Concentrate in Animal Long Bone Healing: An Analysis of Basic Science Evidence. J Orthop Trauma 30: 1-9.

12. Hernigou P, Poignard A, Beaujean F, Rouard H (2005) Percutaneous autologous bone-marrow grafting for nonunions. Influence of the number and concentration of progenitor cells. J Bone Joint Surg Am 87: 1430-1437.

13. Connolly JF, Guse R, Tiedeman J, Dehne R (1991) Autologous marrow injection as a substitute for operative grafting of tibial nonunions. Clin Orthop Relat Res 266: 259-270.

14. Garg NK, Gaur S, Sharma S (1993) Percutaneous autogenous bone marrow grafting in 20 cases of ununited fracture. Acta Orthop Scand 64: 671-672.

15. Healey JH, Zimmerman PA, McDonnell JM, Lane JM (1990) Percutaneous bone marrow grafting of delayed union and nonunion in cancer patients. Clin Orthop Relat Res 1990: 280-285. 